\title{
A Simulation Research on Chaotic Behavior of Parabolic and Elliptic Underwater Acoustic Ray Equations
}

\author{
S.-H. LI ${ }^{a}$ AND X.-J. LI ${ }^{b, *}$ \\ ${ }^{a}$ School of Computer Science \&Technology, Xi'an University of Posts \&Telecommunications, Xi'an 710121, China \\ ${ }^{b}$ Institute of Photonics and Photon-Technology, Northwest University, Xi'an 710069, China
}

(Received February 21, 2012; in final form October 23, 2012)

\begin{abstract}
The chaotic behavior of underwater ray system is studied. Because the parabolic equation is an approximation under small ray angle with respect to horizontal, the elliptic equation system is considered here besides the parabolic system. We pay main attention to the interval of large ray angle. A comparison between these two forms of system is performed. We find that when the ray angle is not large $\left(\theta_{0}=0^{\circ}-18^{\circ}\right)$, the two systems show the same qualitative behavior. However, in interval of large ray angle $\left(\theta_{0} \geq 19^{\circ}\right)$, if the perturbation strength is not very small, e.g. $\delta=0.05$, the parabolic system shows regular motion, while the elliptic system exhibits chaotic behavior in most of this interval except a few quasiperiodic islands studded in the chaotic ocean. Dynamical behaviors of the two systems show surprising difference.
\end{abstract}

DOI: $10.12693 /$ APhysPolA.123.53

PACS: $43.30 .+\mathrm{m}, 05.45 . \mathrm{Pq}$

\section{Introduction}

Chaotic phenomenon in underwater ray system has been discovered years ago [1]. In recent years, research on the ray chaos in field of underwater acoustics is still active. Many important results were reported [2-5]. The ray systems have several different forms such as elliptic equation, parabolic equation, etc. Among them, the parabolic system is widely used because of its simplicity. In ray system, dynamic behavior of the ray depends on the sound speed and the initial conditions. When the sound speed depends only on the depth $z$, the system behavior is regular. On the other hand, if the sound speed depends on the range $r$, the system may exhibit chaotic behavior $[1,2,6,7]$. Similar phenomena also appear in a focusing fiber with range-dependent refractive index $[8,9]$ and a rotor-active magnetic bearing system with a periodically time-varying stiffness [10].

The parabolic equation is an approximation from the elliptic equation under small ray angle with respect to the horizontal $[6,11]$. Previous paper made a conclusion that the parabolic system and the elliptic system exhibit the same qualitative behavior under the periodic perturbation [6]. The study of Ref. [6] was performed within the interval of small ray angle. Other research based on the parabolic system found that the steep rays (with larger ray angle) are more stable than the near-axial rays (with smaller ray angle) [12].

In many cases, e.g. under large initial ray angle, the large ray angle is sure to be met. Then, how does the underwater acoustic ray actually act, particularly in the interval of large ray angle? Because of the small ray angle approximation, we may have some reasons to question the result of the parabolic system in this interval. In this

*corresponding author; e-mail: lixiaojun@nwu.edu.cn paper, the one-way elliptic equation, which is not limited within the interval of small ray angle, is applied to make a more exact solution under large ray angle. This gives a full view on the dynamic behavior of the underwater ray system. The nonlinear dynamic tools such as the Poincaré section, the Lyapunov exponent, correlation dimension are used to describe the chaotic property of the system when the periodic perturbation is added and its strength increases. A comparison between the parabolic system and the one-way elliptic system is performed. The results indicate that if the initial ray angle is not large, these two systems are in good agreement. However, in interval of large ray angle, when the strength of the periodic perturbation is not very small, the results of the two systems are different.

\section{System model}

The sound propagation in the ocean can be described by the ray system under some conditions. One form of the acoustic ray equation consistent with the elliptic (Helmholtz) wave equation is [6]:

$$
\begin{aligned}
& \frac{\mathrm{d} z}{\mathrm{~d} r}=\frac{\partial H}{\partial k_{z}}, \\
& \frac{\mathrm{d} k_{z}}{\mathrm{~d} r}=-\frac{\partial H}{\partial z} .
\end{aligned}
$$

Here $H$ is the Hamiltonian,

$$
H\left(z, k_{z}, r\right)=-k_{r}=-\left[\omega^{2} / c^{2}(z, r)-k_{z}^{2}\right]^{1 / 2},
$$

where $z$ is the depth which is defined positive downwards, $r$ is the range, and $\omega$ is the angular frequency of the sound wave, $c(z, r)$ is the sound speed, $\boldsymbol{k}=\left(k_{z}, k_{r}\right)$ with $|\boldsymbol{k}|=k$ is the wave-number vector. Equation (1) is called one-way elliptic equation.

When the ray angle with respect to the horizontal $\theta$ $\left(\theta=\tan ^{-1}\left(k_{z} / k_{r}\right)\right)$ is small, i.e. $|\theta| \ll 1$, Eq. (1) can be 
reduced to the following form through a special approximation $[6,11]$ :

$$
\begin{aligned}
& \frac{\mathrm{d} z}{\mathrm{~d} r}=\frac{\partial H}{\partial p}, \\
& \frac{\mathrm{d} p}{\mathrm{~d} r}=-\frac{\partial H}{\partial z},
\end{aligned}
$$

where

$$
H(z, p, r)=p^{2} / 2+\left[c(z, r)-c_{0}\right] / c_{0} .
$$

Here $p=\tan \theta$, and $c$ is the reference sound speed. Equation (3) is the parabolic equation. For its simplicity in theoretical analysis and numerical calculation, the parabolic system is widely used in researches on ray systems.

Substituting Eq. (2) into Eq. (1) gives

$$
\begin{aligned}
& \frac{\mathrm{d} z}{\mathrm{~d} r}=k_{z}\left[\omega^{2} / c^{2}(z, r)-k_{z}^{2}\right]^{-1 / 2}, \\
& \frac{\mathrm{d} k_{z}}{\mathrm{~d} r}=-\left[\omega^{2} / c^{2}(z, r)-k_{z}^{2}\right]^{-1 / 2} \frac{\omega^{2}}{c^{3}(z, r)} c_{z}(z, r) .
\end{aligned}
$$

Here $c_{z}(z, r)=\partial c(z, r) / \partial z$.

Substituting Eq. (4) into Eq. (3) gives

$$
\begin{aligned}
& \frac{\mathrm{d} z}{\mathrm{~d} r}=p, \\
& \frac{\mathrm{d} p}{\mathrm{~d} r}=-\frac{1}{c_{0}} c_{z}(z, r) .
\end{aligned}
$$

Above systems, both parabolic equation and elliptic equation, are integrable and thus show regular behavior when the sound speed is independent of range. On the other hand, when the sound speed is range-dependent, generally, the above systems are non-integrable and may exhibit chaotic dynamic behavior $[1,6]$. Here we can see that the sound speed plays an important role in determining the manner of the system behavior. This paper actually bases on an investigation that how the sound speed affects the ray behavior.

The sound speed used in this paper is shown as below [6]:

$$
\begin{aligned}
& c(z, r)=c_{0}\left[1+\varepsilon\left(\mathrm{e}^{-\eta}+\eta-1\right)\right. \\
& \left.\quad+\delta(2 z / B) \mathrm{e}^{-2 z / B} \cos (2 \pi r / \lambda)\right],
\end{aligned}
$$

where $\eta=2\left(z-z_{\text {axis }}\right) / B$ is a scaled depth coordinate, $z_{\text {axis }}$ is the sound channel axis depth, $B$ is the depth scale. In Eq. (7), the first term together with the second term in the square bracket is a range-independent background sound-speed profile, which is the Munk model [13]. The third term in the square bracket represents the periodic range-dependent perturbation. $\varepsilon$ and $\delta$ are coefficients. $\lambda$ is the wavelength of the periodic perturbation, and $\delta$ may be considered as perturbation strength. Then

$$
\begin{gathered}
c_{z}(z, r)=\frac{\partial c(z, r)}{\partial z}=c_{0} \frac{2}{B}\left[\varepsilon\left(1-\mathrm{e}^{-\eta}\right)\right. \\
\left.+\delta \mathrm{e}^{-\frac{2 z}{B}}\left(1-\frac{2 z}{B}\right) \cos (2 \pi r / \lambda)\right] .
\end{gathered}
$$

In order to make a comparison with previous research, values of some parameters and coefficients in the Munk background profile and the periodic range-dependent perturbation are taken the same as they were in previous papers [6]. Here $c_{0}=1.49 \mathrm{~km} / \mathrm{s}, z_{\text {axis }}=1 \mathrm{~km}, B=1 \mathrm{~km}$, $\lambda=10 \mathrm{~km}, \varepsilon=0.0057$.

\section{Numerical calculations and discussions}

In this section, dynamic behaviors of the parabolic system and the elliptic system under the periodic range-dependent perturbation are investigated numerically. A comparison between results of the elliptic system and the parabolic system in the interval of large initial ray angle is performed.

Based on the elliptic system Eq. (5) and the parabolic system Eq. (6), a variety of numerical calculations are performed. The system behavior is studied using some nonlinear dynamic tools such as the Poincare section, the Lyapunov exponent, correlation dimension, etc. The differential equations are solved by means of fourth-order Runge-Kutta method. The sound source is placed at the sound channel axis so the initial depth $z_{0}=1 \mathrm{~km}$. A number of trajectories corresponding to the initial ray angle $\theta_{0}=0^{\circ}, 1^{\circ}, \ldots, 30^{\circ}$, are studied.

The Poincaré sections of the parabolic system and the elliptic system are given in Figs. 1-3. Figures 1-3 are versus the periodic perturbation strength $\delta=0.01, \delta=0.03$, and $\delta=0.05$, respectively. A comparison is made between the two systems here. Parts (a) in Figs. 1-3 are, versus the parabolic system, and parts (b) in these figures versus the elliptic system. We can find in Fig. 1 that, under small strength of the periodic perturbation $(\delta=0.01)$, the two systems show the same qualitative behavior. While in Fig. 3, under a bit larger perturbation strength $(\delta=0.05)$, when the initial ray angle is small, e.g. $\theta_{0}=0^{\circ}-4^{\circ}$, the Poincaré sections of the two system are closed curves. This means that the two systems all exhibit regular behavior. For those trajectories whose initial angles are moderate e.g. $\theta_{0}=5^{\circ}, 6^{\circ}, \ldots$, $18^{\circ}$, the Poincare sections of the two systems appear as the scatter spots, this means that both systems exhibit chaotic behavior. In all, we may conclude that in above interval of initial ray angle, the two systems exhibit the same qualitative behavior. But if we devote our mind into the interval of large initial ray angle, the situations would be quite different. For $\theta_{0} \geq 19^{\circ}$, the parabolic system shows regular motion. However, the elliptic system exhibits chaotic behavior in most of this interval except a few quasiperiodic islands studded in the chaotic ocean.

Evolvement of the difference between the dynamic behaviors of the two systems can be seen in Figs. 1-3. In the elliptic system, some originally regular trajectories with large initial ray angle split into successions of regular orbits at smaller scale. When the perturbation strength increases, the overlap of resonance zone occurs. Therefore, the condition of the KAM theorem begins to break down. Then, the chaos appears [14]. However, in the parabolic system, such overlap of resonance zone does not happen, and trajectories with large initial ray angle remain regular. 


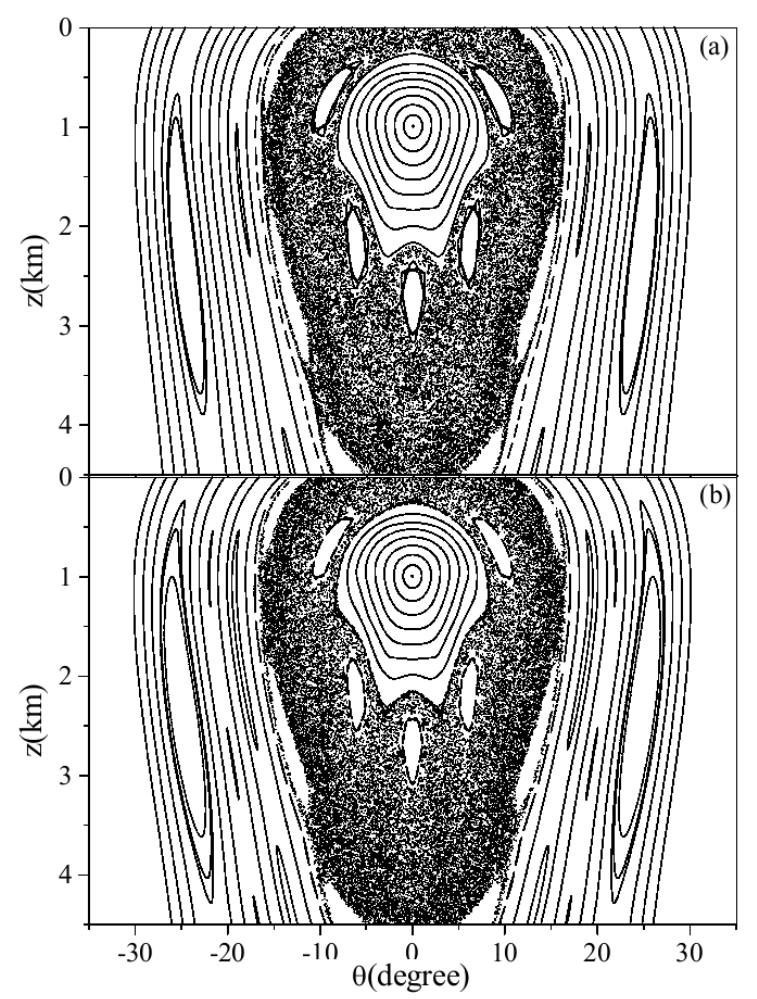

Fig. 1. Poincaré sections of the systems. $\delta=0.01$. (a) Parabolic system, (b) elliptic system.

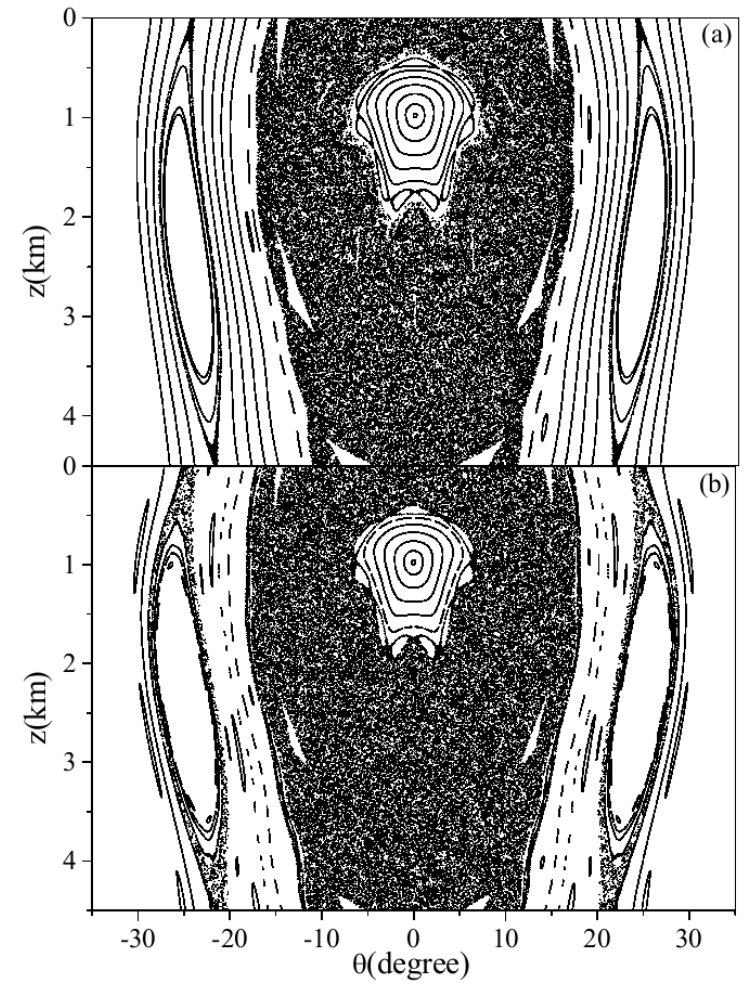

Fig. 2. As in Fig. 1, but for $\delta=0.03$.

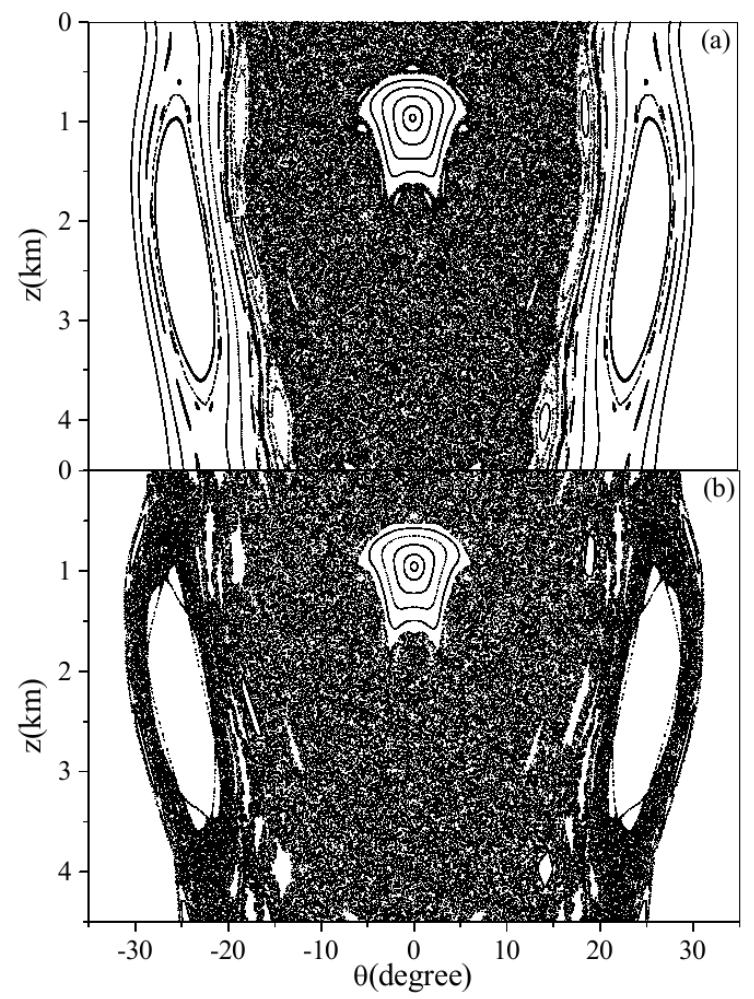

Fig. 3. As in Fig. 1, but for $\delta=0.05$.

Above investigation by means of the Poincare section gives a qualitative view on the difference of the dynamic behavior of the two systems. Further more, we need the way to tell how irregularity of the system behavior evolves. One of such quantitative tools is known as the Lyapunov exponent. The Lyapunov exponent is the average exponential rate of divergence or convergence of neighboring orbits in phase space. Consider a dynamic system

$$
\frac{\mathrm{d} \boldsymbol{X}}{\mathrm{d} t}=F(\boldsymbol{X}),
$$

where $\boldsymbol{X}$ is an $n$-dimensional vector in the state space. In the parabolic ray system which is presented in this paper, $\boldsymbol{X}=(z, p)^{\mathrm{T}}$, and in the elliptic system, $\boldsymbol{X}=\left(z, k_{z}\right)^{\mathrm{T}} \cdot t$ is corresponding to the range $r$. The evolution of the tangent vector $\boldsymbol{W}$ in the tangent space of $\boldsymbol{X}(t)$ is given by the linearization of Eq. (9),

$$
\delta \dot{\boldsymbol{W}}=F_{\boldsymbol{X}}(\boldsymbol{W}) \delta \boldsymbol{W},
$$

where $\boldsymbol{W}$ is an $n$-dimensional eigenvector which represents the evolution of a coordinate system corresponding to the Lyapunov exponent, and $\delta \boldsymbol{W}$ is the significantly small disturbance to $\boldsymbol{W}$. Let $\delta \boldsymbol{W}$ be the $n$-dimensional basis of the tangent space, then the Lyapunov exponents $\lambda_{i}$ are given by [7]:

$$
\lambda_{i}=\lim _{t \rightarrow \infty} \frac{1}{t} \ln \frac{\left\|\delta \boldsymbol{W}_{i}(t)\right\|}{\left\|\delta \boldsymbol{W}_{i}\left(t_{0}\right)\right\|} \quad(i=1,2, \ldots, n),
$$

where $\boldsymbol{W}_{i}(t)$ is the $i$-th basis vector in tangent space, $t_{0}$ is the initial time. 
A system with positive Lyapunov exponent is chaotic, and if all of the Lyapunov exponents of the system are no more than zero, this system is regular. The Lyapunov exponent is calculated by computer numerical method in this paper.

In addition, we choose correlation dimension $\left(D_{2}\right)$ as another quantitative tool to describe the system behavior. Correlation dimension images the spatial shape of the system orbit from view of geometry and topology.

Correlation dimension can be measured through a time series taken from the system: $x\left(t_{1}\right), x\left(t_{2}\right), x\left(t_{3}\right), \ldots$, where $x\left(t_{i}\right) \in R, t_{i}=t_{0}+i \Delta t$. The measurement $x(t)$ in fact represents a projection from full state vector $\boldsymbol{X}(t) \in$ $\boldsymbol{R}^{\mathrm{M}}: \pi: \boldsymbol{R}^{\mathrm{M}} \rightarrow \boldsymbol{R}$. Here the time $t$ corresponds to the range $r$ in the ray system, and the time series $x(t)$ can be depth $z$ or ray angle $\theta$ taken from a single trajectory in the parabolic system (in the elliptic system, it is $k_{z}$ instead of $z$ ).

A vector $\hat{\boldsymbol{X}}(t)$ representing the reconstructed state space is defined: $\hat{\boldsymbol{X}}(t)=[x(t), x(t-\Delta t), \ldots, x(t-(m-$ 1) $\Delta t)]^{\mathrm{T}}$, where $\Delta t$ is the delay time and $m$ is the embedding dimension. Then, correlation dimension can be calculated from correlation integral [15]. Correlation integral can be directly calculated from a finite time series

$$
C(\varepsilon)=\frac{1}{N(N-1)} \sum_{i=1}^{N} \sum_{\substack{j=1 \\ i \neq j}}^{N} \Theta\left(\varepsilon-\left\|\hat{\boldsymbol{X}}_{i}-\hat{\boldsymbol{X}}_{j}\right\|\right),
$$

where

$$
\Theta(x)= \begin{cases}1, & x>0 \\ 0, & x \leq 0\end{cases}
$$

$N$ is size of the time series $\hat{\boldsymbol{X}}(t)$.

Thus, there is the following correlation dimension formula for finite time series for a large enough embedding dimension:

$$
v=\lim _{\varepsilon \rightarrow 0} \lim _{N \rightarrow \infty} \frac{\log C(N, \varepsilon)}{\log \varepsilon} .
$$

The algorithm of calculating correlation dimension according to Eq. (13) converges very slow as $\varepsilon \rightarrow 0$. The efficiency of getting correlation integral is too low. To solve this problem, calculation method of local slope is used

$$
\hat{v}=\frac{\mathrm{d} \log C(N, \varepsilon)}{\mathrm{d} \log \varepsilon}=\frac{\Delta \log C(N, \varepsilon)}{\Delta \log \varepsilon} .
$$

In some region, the curve of local slope $\hat{v}$ is relatively smooth. This region is called linear smooth region where $\hat{v} \approx v$. It should be noticed that only when embedding dimension is big enough, the calculated correlation dimension is the real correlation dimension, that is $v=D_{2}$.

For a quasiperiodic orbit in our two-dimensional system, value of the correlation dimension is 2.0 , and for the chaotic attractor, the correlation dimension is a fraction more than 2.0. The correlation dimension is also calculated numerically with a self-edit computer program here.
The Lyapunov exponents and the correlation dimension are given in Fig. 4 and Fig. 5, respectively. Parts (a) and parts (b) are versus the periodic perturbation strength $\delta=0.01$ and $\delta=0.05$, respectively. The solid line corresponds to the elliptic system and the dashed line to the parabolic system in these two figures. In Fig. 4, only the maximum Lyapunov exponent is given.

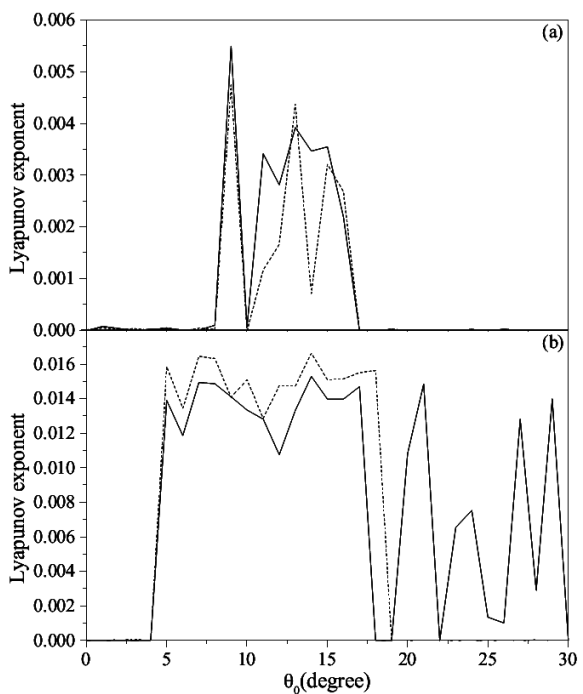

Fig. 4. Lyapunov exponent on the initial ray angle. Solid line: the elliptic system; dashed line: the parabolic system. (a) $\delta=0.01$, (b) $\delta=0.05$.

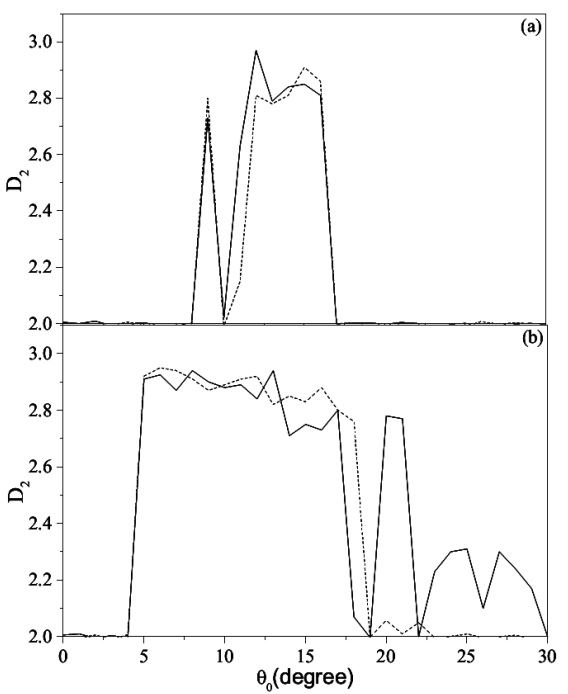

Fig. 5. Correlation dimension on the initial ray angle. Solid line: the elliptic system; dashed line: the parabolic system. (a) $\delta=0.01$, (b) $\delta=0.05$.

It can be found in Fig. 4a and Fig. 5a that the Lyapunov exponent curves and correlation dimension curves of the two systems have identical fashion when the periodic perturbation strength $\delta=0.01$. This indicates that 
the two systems have the same dynamical characteristics under this condition. Then considering Fig. $4 \mathrm{~b}$ and Fig. 5b $(\delta=0.05)$, some differences of chaotic behavior between the two systems would be found. When the initial ray angle is not large, the Lyapunov exponents and correlation dimension of the two systems have identical varying tendency. But when $\theta_{0}$ is large, the curves of the two systems separate. The Lyapunov exponent of the parabolic system turns to decrease to zero (correlation dimension decreases to 2.0), i.e. in this interval, behavior of the parabolic system is regular. However, the behavior of the elliptic system remains chaotic in most of this interval; a few discrete zero values of the Lyapunov exponent (and values 2.0 of the correlation dimension) in this interval correspond to the quasiperiodic islands in Fig. 3b. By the way, it can be noticed that when the initial ray angle is large, irregularity of the parabolic system has a tendency of decrease. Here, irregularity of the elliptic system has a tendency of decrease, too. The difference is that the parabolic system evolves to regular while the elliptic system does not.

\section{Summary}

Chaotic behaviors of the parabolic system and the elliptic system have been investigated with several dynamic tools such as the Poincaré section, the Lyapunov exponent, correlation dimension, etc. A comparison between the two systems was performed. We pay much attention to the interval of large initial ray angle, and find that the dynamic behavior of the parabolic system and the elliptic is quite different from each other in this interval, although they are qualitatively the same in interval of small ray angle. Some trajectories with large initial ray angle in the parabolic system appear in regular fashion, but the trajectories with the same initial conditions in the elliptic system may exhibit chaotic behavior. This difference of dynamic behavior between the two systems comes from the small ray angle approximation of the parabolic system, and the result of the elliptic system may be more reasonable in this interval. The study in this paper enlarges the scope of investigation on ray chaos, and overcomes the limitation that some work based on the parabolic system has.

\section{Acknowledgments}

This work was supported by Natural Research Project (12JK0519) of Education Department of Shaanxi Province, China.

\section{References}

[1] D.R. Palmer, M.G. Brown, F.D. Tappert, H.F. Bezdek, Geophys. Res. Lett. 15, 569 (1988)

[2] T. Bódai, A.J. Fenwick, M. Wiercigroch, J. Sound Vibrat. 324, 850 (2009)

[3] T. Bódai, A.J. Fenwick, M. Wiercigroch, Int. J. Bifurcat. Chaos 18, 1579 (2008)

[4] I.P. Smirnov, A.L. Virovlyansky, G.M. Zaslavsky, J. Acoust. Soc. Am. 117, 1595 (2005)

[5] T. Bódai, A.J. Fenwick, M. Wiercigroch, Int. J. Bifurcat. Chaos 19, 2953 (2009)

[6] K.B. Smith, M.G. Brown, F.D. Tappert, J. Acoust. Soc. Am. 91, 1939 (1992)

[7] X.J. Li, Y. Zhang, G.H. Du, J. Acoust. Soc. Am. 105, $2142(1999)$

[8] X.J. Li, G.H. Du, J. Opt. Soc. Am. B 18, 318 (2001)

[9] Y.A. Li, X.J. Li, J.T. Bai, J. Northwest Univ. (Natural Science Edition) 34, 165 (2004) (in Chinese).

[10] M. Eissa, M. Kamel, H.S. Bauomy, Int. J. Bifurcat. Chaos 21, 195 (2011)

[11] F.D. Tappert, in: Physics, Vol. 70, Wave Propagation and Underwater Acoustics, Eds. J.B. Keller, J.S. Papadakis, Springer-Verlag, New York 1977, p. 224.

[12] K.B. Smith, M.G. Brown, F.D. Tappert, J. Acoust. Soc. Am. 91, 1950 (1992)

[13] W.H. Munk, J. Acoust. Soc. Am. 55, 220 (1974)

[14] J. Yan, J. Acoust. Soc. Am. 94, 2739 (1993)

[15] P. Grassberger, I. Procaccia, Physica D 9, 189 (1983) 